\title{
Discrimination formation as related to the amount of $s^{\Delta}$ training
}

\author{
J. Gilmour Sherman, Frederiek W. Hegge, and Rosemary Pierrel \\ BROWN UNIVERSITY
}

\begin{abstract}
Rats were trained on a two-valued auditory-intensity discrimination. Daily sessions consisted of $1 \mathrm{hr}$. of $\mathrm{S}$ and 1,2 , or $5 \mathrm{hr}$. of $\mathrm{S} \Delta$. Discrimination performance was directly related to the amount of $\mathrm{S}^{\Delta}$ training. Any given amount of $\mathrm{S}^{\Delta}$ exposure was equally effective whether presented under massed or distributed conditions.
\end{abstract}

\section{Problem}

In experiments designed to study the learning of a discrimination using operant conditioning techniques, the organism is exposed to an alternation of a positive and a negative stimulus. The classical procedure in such studies is to associate the negative stimulus ( ${ }^{\Delta}$ ) with periods of extinction and the positive stimulus $\left(\mathrm{S}^{\mathrm{D}}\right)$ with periods of reinforcement. Skinner (1938) pointed out that "since conditioning takes place more rapidly than extinction, we shall presumably need a greater amount of the latter." This assumption has been tacitly accepted in the design of most discrimination experiments, rather than having been systematically studied in terms of the effect of varying amounts of $\mathrm{S}^{\Delta}$. The present study investigates the formation of an auditory intensity discrimination under conditions in which daily training time devoted to $\mathrm{S}^{\mathrm{D}}$ was constant and the time devoted to $\mathrm{S} \Delta$ was varied.

\section{Apparatus}

The apparatus employed has been described in detail elsewhere (Pierrel \& Sherman, 1960). In brief, each unit consisted of a training enclosure which was located within a sound-shielded refrigerator shell and was equipped with a lever (bar). Sound stimuli were presented through a speaker above the rat enclosure. Food pellets were dropped into a dish below the lever. The apparatus was fully automated; ventilation, pellet delivery, changes in sound intensity and the recording of the bar-press responses on counters and cumulative ink recorders were controlled from an adjacent room. Four identical experimental chambers were used.

Two sound intensities, differing by $40 \mathrm{db}$, were employed as the $\mathrm{S}^{\mathrm{D}}$ and the $\mathrm{S} \Delta$ intensities. The stimuli were $4 \mathrm{~K}$ cps tones. The reference signal was $100 \mathrm{db}$ above .0002 dynes $/ \mathrm{cm}^{2}\left(\mathrm{~S}^{\Delta}\right)$. Hereafter, the $\mathrm{S} \Delta$ intensity will be referred to as $0 \mathrm{db}$ and the $\mathrm{S} D$ as $40 \mathrm{db}$ (attenuation from the reference value).

\section{Method}

Prior to the start of the training sessions, the animals were reduced to $80 \%$ of their free-feeding weights and maintained at this value throughout the study. The animals were randomly assigned to three groups: Group I, three animals; Group II, four animals; Group III, three animals.

On the day preceding the start of discrimination training, each animal was placed in an experimental unit in the presence of the intensity which was to serve as $\mathrm{S} D$ $(40 \mathrm{db})$ and allowed to collect 100 regular reinforcements for bar pressing. For the following 13 days discrimination training was carried out (an apparatus failure resulted in the loss of the data for Group III on the last day). The animals all received daily sessions with $1 \mathrm{hr}$. of $\mathrm{S}^{\mathrm{D}}$, the group treatment differing only in the amount of daily session time devoted to $\mathrm{S}^{\Delta}$ : Group I, $1 \mathrm{hr}$.; Group II, $2 \mathrm{hr}$.; Group III, $5 \mathrm{hr}$. This resulted in daily session lengths ranging between 2 and $6 \mathrm{hr}$. among the three groups. The $S \cdot D$ and $S^{\Delta}$ presentations were alternated throughout a session with periods of $\mathrm{S}^{\mathrm{D}}$ ranging from 1 to $3 \mathrm{~min}$. in length. The $\mathrm{S}^{\Delta}$ periods ranged from 1 to $3 \mathrm{~min}$. for Group I, from 2 to $6 \mathrm{~min}$. for Group II and from 5 to $15 \mathrm{~min}$. for Group III. For all groups, reinforcement was programmed to occur irregularly on the average of once a minute during $S^{\prime}$ (VI 1$)$.

\section{Results}

Mean daily discrimination ratios for each group are shown in Fig. 1. The ratios were computed by dividing

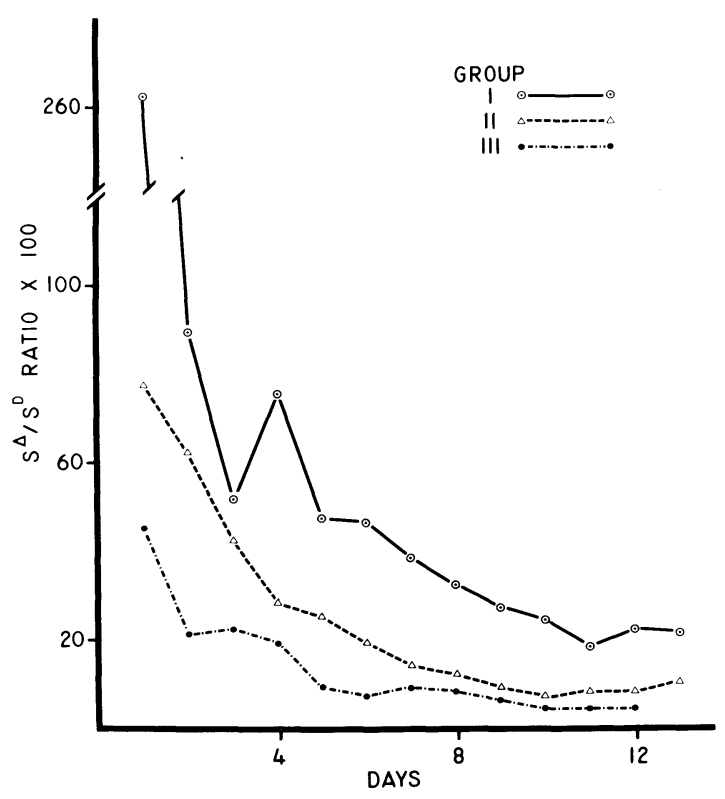

Fig. 1. $\mathrm{S}^{\Delta / \mathrm{S}^{\mathrm{D}}}$ response ratios for each group during 13 days of discrimination training. Each point on the Group II function is the mean of the $S^{\Delta} / S^{D}$ ratios for four Ss. The points on the Group I and Group III functions are means derived from three Ss in each group. 


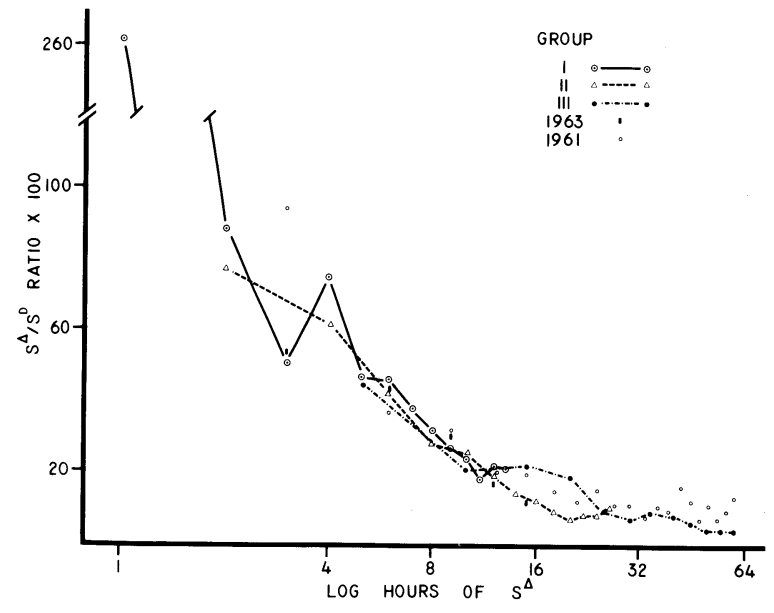

Fig. 2. $\mathrm{S}^{\Delta} / \mathrm{S}^{\mathrm{D}}$ response ratios from Fig. 1 as a function of hours of $\mathrm{S}^{\Delta}$ exposure. Data marked " 1961 ", and "1963", are taken from earlier studies (see text).

the number of responses in $S \Delta$ by the number of responses in $\mathrm{S}^{\mathrm{D}}$. (The $\mathrm{S}^{\Delta}$ response totals for each animal in Groups II and III were first divided by the appropriate constant to correct for the disproportionate amount of session time devoted to $S \Delta$.) The group mean ratios throughout training were inversely related to the amount of daily $S^{\Delta}$ exposure. Clearly, the discriminations are improved by increasing the amount of $S^{\Delta}$. This finding confirms Skinner's earlier speculation as to the significance of extinction.

In Fig. 2 the data from Fig. 1 has been plotted to show the discrimination ratios as a function of hours of $\mathrm{S} \Delta$ exposure. Also shown in Fig. 2 are discrimination data from two previous studies in which animals were also exposed to a $40 \mathrm{dbS}-\mathrm{S}^{\Delta}$ intensity difference. In both studies the data were obtained under conditions similar to those employed in the present study with the exception that $\mathrm{S} \Delta$ was presented for $3 \mathrm{hr}$. in each daily session. The points indicated as " 1963 " are the mean $\mathrm{S} \Delta / \mathrm{S} D$ ratios of Group I from Pierrel et al., (1963). The points indicated as " 1961 " are the mean discrimination ratios of Group I from Sherman and Pierrel (1961).

\section{Conelusions}

When the discrimination ratios are considered in terms of hours of $S^{\Delta}$ exposure, there appear to be no systematic differences between groups. Although for any given number of hours of $\mathrm{S}^{\Delta}$ training, the total number of hours of $\mathrm{S}^{\mathrm{D}}$ presentation differs considerably among the three groups, this appears to make very little difference. Within the range tested, there is no apparent effect produced by massed or distributed exposure to $\mathrm{S} \Delta$ conditions. In other words, this suggests that a given amount of $\mathrm{S}^{\Delta}$ training has a constant effect with respect to discrimination formation.

\section{References}

PIERREL, R., \& SHERMAN, J.G.Generalization of auditory intensity following discrimination training. J. exp. Anal. Beh., 1960, 3, 313. PIERREL, R., SHERMAN, J. G., \& FISCHMAN, M. W. Generalization of auditory intensity as a function of small amounts of discrimination training. J. exp. Anal. Beh., 1963, 6, 545.

SHERMAN, J. G., \& PIERREL, R. Generalization of auditory intensity as a function of the amount of discrimination training. J.exp. Anal. Beh., 1961, 4, 237.

SKINNER, B. F. The behavior of organisms. New York: AppletonCentury-Crofts, 1938. P. 183.

\section{Note}

1. This study was conducted as a part of a research program under United States Public Health Service Grant MY-5757. 